\title{
Clinical utility gene card for: Angelman Syndrome
}

\author{
Karin Buiting ${ }^{\star, 1}$, Jill Clayton-Smith ${ }^{2}$, Daniel J Driscoll ${ }^{3}$, Gabriele Gillessen-Kaesbach ${ }^{4}$, Deniz Kanber ${ }^{1}$, \\ Eberhard Schwinger ${ }^{4}$, Charles Williams ${ }^{3}$ and Bernhard Horsthemke ${ }^{1}$
}

European Journal of Human Genetics (2015) 23, doi:10.1038/ejhg.2014.93; published online 4 June 2014

\section{DISEASE CHARACTERISTICS \\ 1.1 Name of the disease (synonyms) \\ Angelman Syndrome (AS).}

\subsection{OMIM\# of the disease}

105830.

1.3 Name of the analysed genes or DNA/chromosome segments UBE3A/\#15q11-q13.

\subsection{OMIM\# of the gene(s)}

601623.

\subsection{Mutational spectrum}

75\% Maternal deletion 15q11-q13.

1-2\% Paternal uniparental disomy (upd(15)pat).

$3 \%$ Imprinting defect.

$5-10 \%$ Variants in the UBE3A gene.

$10-15 \%$ Unknown (It is important to exclude differential diagnoses in these cases as there is phenotypic overlap with several other genetic disorders).

Data on this disease (gene variants/phenotype) can be found in the public database Decipher (https://decipher.sanger.ac.uk).

\subsection{Analytical methods}

The most sensitive approach in diagnosing AS is to study the DNA methylation pattern within the imprinted region 15q11-q13, in particular at the differentially methylated SNURF-SNRPN exon1/ promoter region. Normal individuals have an unmethylated paternal allele and a methylated maternal allele. In patients with AS who have a de novo maternal deletion of $15 \mathrm{q} 11-\mathrm{q} 13$, upd(15)pat or an imprinting defect only a paternal unmethylated allele can be detected. Patients with a $U B E 3 A$ variant have a normal methylation pattern. The most widely adopted methods for methylation analysis are the methylationspecific (MS)-PCR and the MS-MLPA (Multiplex Ligation Dependent Probe Amplification).

MS-PCR can detect methylation changes due to a $15 \mathrm{q} 11-\mathrm{q} 13$ deletion, upd(15)pat and an imprinting defect, but cannot distinguish between these underlying causes. Determination of the underlying cause is not possible. The same is true for other methylation analyses, including PCR following restriction digestion with a methylationsensitive enzyme, melt-curve analysis and pyrosequencing, but these are rarely used. A follow-up testing that specifically interrogates each of the different types of molecular defects is necessary. This can be done by FISH or DNA microarray analysis to detect 15q11-q13 deletions, or by microsatellite analysis which can distinguish between deletions, upd(15)pat (uniparental inheritance of chromosomes 15) and imprinting defects (biparental inheritance of chromosomes 15).

MS-MLPA has the advantage that it can simultaneously detect changes in copy number and DNA methylation in a semiquantitative manner. Therefore, MS-MLPA can not only detect the presence of a methylation defect due to a 15q11-q13 deletion, a upd(15)pat or an imprinting defect, but it can also distinguish 15q11-q13 and imprinting centre (IC) deletions. Thus, MS-MLPA can determine the molecular defect directly in more than $75 \%$ of patients. As the MS-MLPA cannot distinguish between upd(15)pat and a non-IC deletion imprinting defect, further testing by microsatellite analysis is needed.

In patients who have a normal methylation pattern, but clinical features suggestive of AS, a UBE3A screening for variants should be performed.

A more detailed and expanded overview about molecular testing strategies in AS is given in the 'Best Practice guidelines for the molecular analysis of Prader-Willi and Angelman Syndromes.'

\subsection{Analytical validation}

Parallel analysis of positive and negative controls.

1.8 Estimated frequency of the disease (Incidence at birth ('birth prevalence') or population prevalence. If known to be variable between ethnic groups, please report):

Prevalence at birth: approximately 1:12000 to $1: 20000 .^{2}$

\subsection{Diagnostic setting}

\begin{tabular}{lll}
\hline & Yes & No \\
A. (Differential) diagnostics & $\bigotimes$ & $\square$ \\
B. Predictive testing & $\square$ & $\square$ \\
C. Risk assessment in relatives & $\Downarrow$ & $\square$ \\
D. Prenatal & $\Downarrow$ & $\square$
\end{tabular}

Comment: In AS cases with a 15q11-q13 de novo deletion or upd(15)pat, the recurrence risk is very low if parental chromosomes

${ }^{1}$ Institute of Human Genetics, University Hospital Essen, University Duisburg-Essen, Essen, Germany; ${ }^{2}$ Manchester Centre For Genomic Medicine, Manchester Academic Health Sciences Centre, St Mary's Hospital, Manchester, UK; ${ }^{3}$ Division of Genetics and Metabolism, Department of Pediatrics, University of Florida College of Medicine, Gainesville, FL, USA; ${ }^{4}$ Institut für Humangenetik, Universität zu Lübeck, Lübeck, Germany

*Correspondence: Dr K Buiting, Institute of Human Genetics, University Hospital Essen, University Duisburg-Essen, Hufelandstrasse 55, Essen 45122, Germany. Tel: +49 201 7234555; Fax: +49 201 7235900; E-mail: karin.buiting@uni-due.de

Received 29 January 2014; revised 16 April 2014; accepted 30 April 2014 
are normal. In rare cases with a de novo deletion $(<1 \%)$, a balanced translocation in the mother involving chromosome 15 confers a high recurrence risk and should be excluded by FISH. In rare cases with a upd(15)pat, a Robertsonian translocation in one of the parents could increase the recurrence risk and should be ruled out by cytogenetic analysis. Patients with an imprinting defect have a maternal and a paternal chromosome 15, but the maternal chromosome 15 carries a paternal imprint. The majority of patients with AS and an imprinting defect are sporadic cases without any detectable variants at the DNA sequence level. Imprinting defects of this type usually do not show familial recurrence, and therefore, appear to be associated with a low recurrence risk. However, approximately $10-15 \%$ of patients with an imprinting defect have a microdeletion of the chromosome 15 IC. In the majority of these cases, the IC deletion is found to be a familial variant associated with a 50\% recurrence risk. If the underlying cause is a maternally inherited $U B E 3 A$ variant, the recurrence risk is $50 \%$ if the mother is a carrier of the variant. In other cases, the IC deletion or the UBE3A variant arises de novo (low recurrence risk) or is a consequence of a germline mosaicism in the mother (increased recurrence risk). ${ }^{3}$

\section{TEST CHARACTERISTICS}

\begin{tabular}{|c|c|c|c|c|}
\hline & \multicolumn{2}{|c|}{ Genotype or disease } & \multirow{2}{*}{$\begin{array}{l}\text { A: True positives } \\
\text { B: False positives }\end{array}$} & \multirow{2}{*}{$\begin{array}{l}\text { C: False negative } \\
\text { D: True negative }\end{array}$} \\
\hline & Present & Absent & & \\
\hline \multicolumn{5}{|l|}{ Test } \\
\hline \multirow[t]{2}{*}{ Positive } & $A$ & $B$ & Sensitivity: & $A /(A+C)$ \\
\hline & & & Specificity: & $D /(D+B)$ \\
\hline \multirow[t]{2}{*}{ Negative } & C & $D$ & Positive predictive value: & $A /(A+B)$ \\
\hline & & & Negative predictive value: & $D /(C+D)$ \\
\hline
\end{tabular}

\subsection{Analytical sensitivity}

(proportion of positive tests if the genotype is present)

Maternal deletion 15q11-q13: nearly 100\%.

Paternal uniparental disomy 15: nearly 100\%.

Imprinting defect: nearly 100\% (low levels of mosaicism might not be detected).

UBE3A variants: nearly $100 \%$.

Chromosomal aberrations: depends on the method used.

\subsection{Analytical specificity}

(proportion of negative tests if the genotype is not present)

Nearly $100 \%$.

\subsection{Clinical sensitivity}

(proportion of positive tests if the disease is present)

The clinical sensitivity can be dependent on variable factors such as age or family history. In such cases a general statement should be given, even if a quantification can only be made case by case.

Nearly $85-90 \%$. In $10-15 \%$ of patients with a clinical diagnosis of AS, no deletion, uniparental disomy, an imprinting defect or an $U B E 3 A$ variant is detected and therefore the molecular defect is unknown.

\subsection{Clinical specificity}

(proportion of negative tests if the disease is not present)

The clinical specificity can be dependent on variable factors such as age or family history. In such cases a general statement should be given, even if a quantification can only be made case by case. Nearly $100 \%$.

\subsection{Positive clinical predictive value}

(life time risk to develop the disease if the test is positive)

The risk to develop the disease if the test is positive is $100 \%$, because all known molecular defects lead to the phenotype.

\subsection{Negative clinical predictive value}

(probability not to develop the disease if the test is negative)

Assume an increased risk based on family history for a non-affected person. Allelic and locus heterogeneity may need to be considered.

Index case in that family had been tested:

If the index case had been tested negative the probability for the non-affected individual not to develop the disease is nearly $100 \%$. Index case in that family had not been tested:

Can only be clarified through analysis of the non-affected individual.

\section{CLINICAL UTILITY}

3.1 (Differential) diagnostics: The tested person is clinically affected

(To be answered if in 1.9 ' $\mathrm{A}$ ' was marked)

\subsubsection{Can a diagnosis be made other than through a genetic test?}

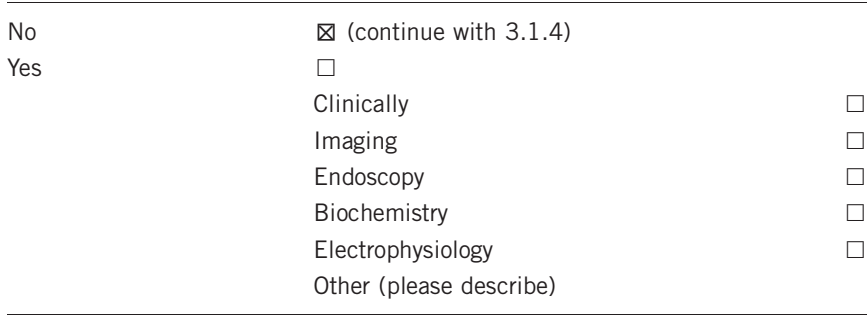

Though the clinical phenotype is characteristic, the significant clinical overlap with the disorders mentioned below means that diagnosis can only be secure when confirmed molecularly. ${ }^{4}$

Conditions most likely to mimic AS

Pitt-Hopkins syndrome (TCF4)

Christianson syndrome (SLC9A6)

Mowat-Wilson syndrome (ZEB2)

Typical Rett syndrome (MECP2)

Other conditions that can also mimic AS

Prader-Willi syndrome (at $<2$ years of age)

22q13.3 deletion, Phelan-McDermid syndrome

2q23.1 deletion, MBD5 haploinsufficiency syndrome

17q21.31 deletion, KANSL1 haploinsufficiency (Koolen-de Vries) syndrome

Kleefstra syndrome (9q34.3 deletion, EHMT1 haploinsufficiency)

HERC2 deficiency syndrome

Adenylosuccinase deficiency

FOXG1 haploinsufficiency syndrome

STXBP1 haploinsufficiency syndrome

MECP2 duplication

$M E F 2 C$ haploinsufficiency syndrome

Alpha-thalassemia/intellectual disability syndrome (ATRX)

3.1.2 Describe the burden of alternative diagnostic methods to the patient

Not applicable. 
3.1.3 How is the cost effectiveness of alternative diagnostic methods to be judged?

Not applicable.

3.1.4 Will disease management be influenced by the result of a genetic test?

\begin{tabular}{ll} 
No & $\bigotimes$ \\
Yes & $\square$ \\
& Therapy (please describe) \\
& Prognosis (please describe) \\
& Management (please describe) \\
\hline
\end{tabular}

3.2 Predictive setting: the tested person is clinically unaffected but carries an increased risk based on family history

(To be answered if in 1.9 'B' was marked)

3.2.1 Will the result of a genetic test influence lifestyle and prevention?

If the test result is positive (please describe).

If the test result is negative (please describe).

3.2.2 Which options in view of lifestyle and prevention does a person at-risk have if no genetic test has been done (please describe)?

Not applicable.

3.3 Genetic risk assessment in family members of a diseased person (To be answered if in $1.9^{~ '} \mathrm{C}$ ' was marked)

\subsubsection{Does the result of a genetic test resolve the genetic situation in} that family?

Yes. Identification of the precise molecular mechanism underlying AS in an individual case will enable accurate identification of at-risk individuals within the family and facilitate accurate genetic counselling. If a UBE3A variant, an imprinting centre deletion or a translocation involving $15 \mathrm{q} 11-13$ is identified, then the mother of the affected case and other family members at risk can be tested to determine the carrier status, although because Angelman Syndrome is an imprinted disorder, these individuals will not be at risk of developing symptoms.

3.3.2 Can a genetic test in the index patient save genetic or other tests in family members?

No.
3.3.3 Does a positive genetic test result in the index patient enable a predictive test in a family member?

Not applicable.

\subsection{Prenatal diagnosis}

(To be answered if in 1.9 ' $\mathrm{D}$ ' was marked)

3.4.1 Does a positive genetic test result in the index patient enable a prenatal diagnosis?

Yes. Testing for a known familial variant can be performed on fetal DNA.

\section{IF APPLICABLE, FURTHER CONSEQUENCES OF TESTING}

Please assume that the result of a genetic test has no immediate medical consequences. Is there any evidence that a genetic test is nevertheless useful for the patient or his/her relatives? (Please describe).

Parents will finally know the cause of the disease. No need for further diagnostic investigations, particularly those to exclude the syndromes mentioned above. A positive molecular diagnosis may confer eligibility for participation in clinical trials and research studies. Moreover, the testing is important for other family members regarding the recurrence of the disease.

\section{CONFLICT OF INTEREST}

The authors declare no conflict of interest.

\section{ACKNOWLEDGEMENTS}

This work was supported by EuroGentest2 (Unit 2: 'Genetic testing as part of health care'), a Coordination Action under FP7 (Grant Agreement Number 261469) and the European Society of Human Genetics. KB, GG-K and BH are supported by the German Ministry of education and sciences BMBF (Network 'Imprinting Diseases', 01GM1114), GG-K and BH are members of the COST Action BM1208. JC-S's work on diagnostic testing in AS and related disorders is supported by Action Medical Research SP4555.

1 Ramsden SC, Clayton-Smith J, Birch R, Buiting K: Practice guidelines for the molecular analysis of Prader-Willi and Angelman syndromes. BMC Med Genet 2010; 11: 11-70. 2 Dagli A, Buiting K, Williams CA: Molecular and clinical aspects of Angelman syndrome. Mol Syndromol 2012; 2: 100-112.

3 Buiting K: Prader-Willi syndrome and Angelman syndrome. Am J Med Genet Part C 2010; 154C: 365-376.

4 Tan W-H, Bird LM, Thibert RL, Williams CA: If not Angelman, what is it? A review of Angelman-like syndromes. Am J Med Genet Part A 2013; 9999: 1-18. 